\title{
Expression of Concern
}

\section{HMGB1 links chronic liver injury to progenitor responses and hepatocarcinogenesis}

Celine Hernandez, Peter Huebener, Jean-Philippe Pradere, Daniel J. Antoine, Richard A. Friedman, and Robert F. Schwabe

Original citation: J Clin Invest. 2018;128(6):2436-2451. https://doi.org/10.1172/JCI91786.

Citation for this expression of concern: J Clin Invest. 2019;129(4):1803. https://doi.org/10.1172/JCI128261.

An investigative committee at the University of Liverpool recently identified evidence of data fabrication relating to the mass spectrometry data contributed by Daniel J. Antoine, shown in Figures 3B and 5A of this paper. The Editorial Board is issuing this Expression of Concern to alert readers to this problem. No issues have been raised in regard to any of the other data in this manuscript.

\section{Corrigendum}

\section{HMGB1 links chronic liver injury to progenitor responses and hepatocarcinogenesis}

Celine Hernandez, Peter Huebener, Jean-Philippe Pradere, Daniel J. Antoine, Richard A. Friedman, and Robert F. Schwabe

Original citation: J Clin Invest. 2018;128(6):2436-2451. https://doi.org/10.1172/JCI91786.

Citation for this corrigendum: J Clin Invest. 2019;129(4):1803. https://doi.org/10.1172/JCI128262.

The Editors posted an Expression of Concern for this article following notification that an investigative committee at the University of Liverpool had data integrity concerns regarding the mass spectrometry data contributed by Daniel J. Antoine, shown in Figures $3 \mathrm{~B}$ and $5 \mathrm{~A}$ of this paper. The authors have also identified errors in the figure labels for Figures $5 \mathrm{H}$ and $6 \mathrm{D}$. The authors have provided a corrected version of this article and a description of changes below.

In our published work, we reported that hepatocyte-derived HMGB1 links cell death to progenitor responses and hepatocarcinogenesis in chronic liver disease (CLD). We based our conclusions on extensive animal studies, including models of chemically or genetically induced chronic cell death in mice with liver-specific knockouts of HMGB1. While hepatocyte-specific deletion of HMGB1 did not significantly alter hepatic injury levels, inflammation, fibrosis, and regeneration, it reduced hepatocyte metaplasia and ductular/progenitor expansion. Passively released HMGB1 promoted ductular reaction in a nonautonomous fashion through RAGE, but not TLR4 or TLR9, as demonstrated by in vivo studies in mice harboring global receptor knockout and further confirmed by in vitro studies using a bipotential liver progenitor cell line. Mice with liver-specific deletion of HMGB1 displayed reduced HCC development in 3 models of chronic hepatocellular death, but not in a model lacking chronic liver injury, further supporting the role of HMGB1 as a DAMP. Similarly to what we observed for CLD, we found that HMGB1 promoted a progenitor signature within tumors, a key feature of HCC aggressiveness.

Figures 3B and 5A show data from an analysis of HMGB1 isoforms by mass spectrometry undertaken in the laboratory of Daniel J. Antoine. In September 2018, we learned that these data were compromised. We contacted the journal, and the Editorial Board agreed to publish an updated online version of the article. In the corrected version, all conclusions based on Figures 3B and 5A have been removed as has reference 78, and the journal has published an online version of the original article with the unreliable statements crossed out and the modified text highlighted in red (Supplemental File, Redaction). We believe that the major conclusions of the study are accurate and that the corrected paper is reliable.

In addition, the labels for HMGB1 and anti-RAGE, anti-TLR4, and the TLR9 inhibitor were accidentally inverted in Figure 5H and have now been corrected. In Figure 6D, the image for the red channel, but not the image for the green channel or the merged image for the wild-type mice, was inadvertently duplicated and has now been replaced with the correct microphotograph. 